\title{
Internal Jugular Vein
}

National Cancer Institute

\section{Source}

National Cancer Institute. Internal Jugular Vein. NCl Thesaurus. Code C32849.

A continuation of the sigmoid sinus of the dura mater; joins the subclavian vein to form the brachiocephalic vein. 